\title{
Bild- und Videokompression
}

\author{
Dietmar Saupe und Raouf Hamzaoui, Universität Konstanz
}

Der Science Fiction Autor Arthur C. Clarke hat einmal festgestellt, dass eine sich erfolgreich entwickelnde Technologie schließlich nicht mehr von Magie unterscheidbar ist. Im Jahr 1921 gelang es Harry G. Bartholomew und Maynard D. McFarlane Photographien zu digitalisieren und erstmals über ein transatlantisches Telegraphiekabel zwischen London und New York hin und her zu schicken. Was damals eine sensationelle aufwändige Ingenieurleistung war und bis zu drei Stunden Übertragungszeit pro Bild erforderte, ist heute alltäglich und blitzschnell. Es genügt, einen Rechner ans Internet anzuschließen, um Bilder und Videos weltweit zu versenden. Dass dabei die digitalen Daten komprimiert und speziell für die Übertragung codiert werden, bleibt im Hintergrund und ist ein Teil der von Clarke beobachteten scheinbaren Magie einer weit fortgeschrittenen Technologie. In diesem Themenheft sehen wir hinter die Kulissen und bieten eine in sich abgerundete Darstellung der Bild- und Videokompression in sechs ausgewählten Einzelbeiträgen. Dabei behandeln wir grundlegende Methoden und Verfahren, technologische Trends, neuartige Anwendungen, Fragen der Standardisierung und geben einen Rückblick auf die Geschichte der Videocodierung in den letzten vier Jahrzehnten.

In dem ersten Beitrag dieses Hefts gibt Kai Uwe Barthel eine elementare Einführung in grundlegende Begriffe und Verfahren der Bildkompression und kommt dann zu Details für die so genannte verlustfreie Bildkompression. Bei der verlustfreien Bildkompression kann das digitale Bild beim Decoder per- fekt rekonstruiert werden. Der Coder führt eine Reduktion der in dem Originalbild enthaltenen Redundanz in Form einer Bildtransformation oder einer Pixelwertprädiktion durch und reduziert den Speicherbedarf weiter durch eine Entropiecodierung. Erfahrungsgemäß lassen sich „normale“ Grauwertbilder mit einem Kompressionsfaktor um 2-3 verlustfrei codieren. Wird jedoch vor der Entropiecodierung eine nicht-umkehrbare Quantisierung der $\mathrm{zu}$ codierenden Zahlen durchgeführt, so ergeben sich wesentlich größere Kompressionsfaktoren. Allerdings kann der Decoder das Originalbild nicht mehr ohne Rekonstruktionsfehler berechnen, man spricht deshalb in diesem Fall von verlustbehafteter Bildkompression.

Seit 1992 ist JPEG ein ISOITU Standard für die verlustbehaftete Bildkompression. JPEG codiert ein Bild in drei Schritten: (1) Das Bild wird in Blöcke unterteilt, die durch die zweidimensionale diskrete Cosinus Transformation (DCT) in den Frequenzraum abgebildet werden. (2) Die DCT-Koeffizienten werden quantisiert. Dabei werden Koeffizienten, die hohen Frequenzen entsprechen auf null gesetzt. (3) Die quantisierten DCT-Koeffizienten werden verlustfrei codiert. James Fowler stellt eine Reihe von Verfahren vor, die die Wavelet-Transformation anstelle der DCT verwenden. Diese Wavelet-basierten Verfahren ermöglichen eine bessere Bildqualität bei gleicher Kompressionsrate und haben die Eigenschaft, dass im Wesentlichen schon jeder Präfix einen Bildcode für die entsprechende Bitrate darstellt und am Decoder eine Approximation des Original- bildes liefert, dessen Qualität mit der Länge des Präfixes wächst. Die Güte solcher Verfahren hat dazu geführt, dass in dem neuen Standard JPEG2000 für verlustbehaftete Bildkompression eine Wavelettransformation anstelle der traditionellen DCT eingesetzt wird.

Die weiteren vier Beiträge des Hefts behandeln die Kompression von Bildsequenzen. Der Aufsatz von Hans-Georg Musmann und Reiner Lippmann liefert eine Darstellung der Entwicklung von Verfahren zur Videocodierung über den gesamten relevanten Zeitraum der letzten vier Jahrzehnte. Wichtige Meilensteine auf dem langen Weg zu den ersten internationalen Standards sind zum Beispiel Methoden zur IntraframeCodierung bei der die Redundanz aufeinander folgender Bilder ausgenutzt wird. Insbesondere ist hier die bewegungskompensierte Bildzu-Bild-Prädiktion zu nennen, bei der sich im Video bewegende Objekte erkannt werden und zur Vorhersage von Teilen zukünftiger Bilder verwendet werden. Die Technologie führte zu einer für den Laien verwirrenden Vielfalt von Standards, die in dem Artikel übersichtlich dargestellt werden. Schließlich werden aktuelle Entwicklungen und Perspektiven für die Zukunft besprochen, die z. B. die Qualität von Videoübertragungen in schmalbandigen Mobilfunkkanälen verbessern werden.

Der Beitrag von Thomas Sikora handelt von dem letzten MPEG Standard zu Videokompression, MPEG-4. In diesem Standard von 1999 wird neben einer effizienten Kompression für die Übertragung in gestörten Kanälen und mit variabler Bandbreite auch eine Interaktivität 
mit Ton- und Bildinhalten unterstützt. Nutzer können inhaltsbezogene Objekte aus der Videosequenz extrahieren und in andere Anwendungsprogramme zur weiteren Bearbeitung kopieren. Auch eine Integration von 3D-Objekten aus der Computergrafik ist vorgesehen.

Die oben schon angesprochene Bewegungskompensation ist ein wichtiger Bestandteil der erfolgreichsten Videokompressionsverfahren, wie etwa MPEG-2, MPEG-4 und H.263. Sie dient dazu, die zeitliche Redundanz von Bildsequenzen auszunützen. Statt einen Bildblock zu codieren, wird die Differenz zwischen dem Block und seiner Prädiktion codiert. Dabei unterscheidet man P-Bilder, wo die Blöcke mit Referenz auf ein vergangenes Bild codiert werden und B-Bilder, wo sie mit Referenz auf ein vergangenes und ein zukünftiges Bild codiert werden. Der Aufsatz von Bernd Girod und Markus Flierl zeigt wie der Einsatz von weiteren Referenzbildern (zum Beispiel fünf vergangene Referenzbilder für ein P-Bild) die Methode signifikant verbessern kann.

Der Artikel von Thomas Stockhammer und Michael Mecking be- schäftigt sich schließlich mit der Videoübertragung für drahtlose Endgeräte, wie sie in Mobilfunksystemen der dritten Generation (UMTS) vorkommt. Solche Systeme sind gekennzeichnet durch eine beschränkte Sendeleistung und Bandbreite. Neben den Eigenschaften mobiler Übertragungskanäle werden Kompressionsaspekte, Transportprotokolle und Fehlerschutzverfahren für verschiedene mobile Anwendungen (Multimedia Messaging Services, Streaming, VideoTelefonie) besprochen.

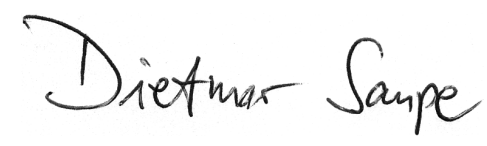
Dietmar Saupe
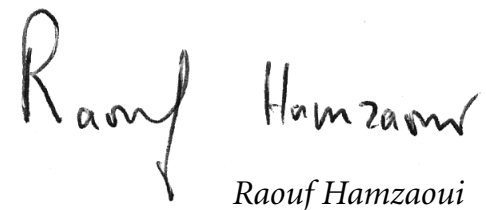

Raouf Hamzaoui

Prof. Dr. Dietmar Saupe promovierte 1982 in Mathematik an der Universität Bremen. Er war Visiting Assistant Professor an der University of California von 1985 bis
1987, Hochschulassistent im Fachbereich Mathematik der Universität Bremen von 1987 bis 1993, anschließend nach der Habilitation in Mathematik im Jahr 1993 Professor für Informatik an den Universitäten in Freiburg (1993-1998), Leipzig (1998-2002) und Konstanz (ab 2002). Seine gegenwärtigen Forschungsinteressen liegen in der Bildverarbeitung, Computergrafik und Visualisierung. Dietmar Saupe ist Sprecher des neugegründeten Fachbereichs Graphische Datenverarbeitung der Gesellschaft für Informatik.

Adresse: Universität Konstanz, Fachbereich Informatik und Informationswissenschaft, Fach M697, D-78457 Konstanz, Tel.: +49 (7531) 88-4220, Fax: -4715. E-Mail: saupe@inf.uni-konstanz.de

Dr. Raouf Hamzaoui promovierte 1997 im Institut für Informatik der AlbertLudwigs-Universität Freiburg, wo er bis 1998 als wissenschaftlicher Mitarbeiter tätig war. Von 1998 bis 2002 war er am Institut für Informatik der Universität Leipzig und seitdem ist er wissenschaftlicher Assistent an der Universität Konstanz. Seine Forschungsinteressen gelten vor allem der Bildkompression und -übertragung. Adresse: Universität Konstanz, Fachbereich Informatik und Informationswissenschaft, Fach M697, D-78457 Konstanz. E-Mail:

hamzaoui@inf.uni-konstanz.de 